\title{
The Greater Zurich Area is Well-positioned in the Life Sciences in Switzerland and Europe
}

\author{
Mario Jenni*
}

\begin{abstract}
The Greater Zurich Area has become a hub for the life sciences industry in recent years, with aboveaverage numbers of medtech companies and brisk spin-off activities from the Zurich universities. The spin-offs have established themselves near their scientific origins, in incubators and parks where they have access to functional and affordable laboratory infrastructures. Numerous cluster initiatives and networks foster exchanges between start-ups, established companies and academia. An international regional comparison conducted within the framework of the EU HealthTIES project gave the region of Zurich a good score in the life sciences area. The detailed comparison shows that Zurich is strong in the academic field and the existing companies are well-positioned. However, weaknesses are evident in the field of clinical research and in the interface between academia and industry. The infrastructure offerings in the Greater Zurich Area for life sciences companies and for exchanges between academia and industry will be further expanded in the coming years. Whether the high growth rate of the industry in the Greater Zurich Area can be maintained or even increased depends on the economy and other factors, but also on the political environment.
\end{abstract}

Keywords: Incubator · Life sciences · Science park · Start-ups · Zurich

\section{Localization of Life Sciences Start- ups in Switzerland}

The term 'life sciences industry in Switzerland' is traditionally associated with the region of Basel, the location of the pharmaceutical groups Novartis and Roche. In addition to the pharmaceutical industry, the industries in the fields of agrochemicals, biotechnology and medical technology are also typically combined under the term 'life sciences'. In the field of medical technology, the Swiss midlands from Geneva to St. Gallen have a high density of companies. The Canton of Zurich has therefore developed into an industry center in recent years and has become the national leader according to the number of employees in this field. ${ }^{[1]}$ The biotech companies in Switzerland are less widely distributed, and are concentrated in centers in the Lake Geneva region (Arc lémanique),

${ }^{*}$ Correspondence: M. Jenn

Co-founder \& Managing Director

BIO-TECHNOPARK Schlieren-Zurich

Wagistrasse 23

$\mathrm{CH}-8952$ Schlieren

Tel.: +4144730 8329

E-mail: mario.jenni@bio-technopark.ch
Bern, Basel, Ticino and in the Greater Zurich Area. ${ }^{[2]}$ The majority of biotech companies that conduct research and development are spin-offs from universities. Experience has shown that these companies establish themselves near their scientific origins, generally in places where they find the required laboratory infrastructure.

\section{Life Sciences Innovation Centers, Business Incubators and Technology Parks in the Greater Zurich Area (Agglomeration of Zurich and Eastern Switzerland)}

Every year, the Swiss Federal Institute of Technology Zurich (ETH Zurich) and the University of Zurich register a considerable number of new spin-offs in the field of life sciences. That is not surprising, but according to the Academic Ranking of World Universities (Shanghai Ranking) 2014, the ETH Zurich ranks 8th in natural sciences, and the University of Zurich is 25th in the field of life sciences. ${ }^{[3]}$ Additionally, the research emphases in life sciences at both universities are very complementary and there are synergies used in scientific centers of excellence (Centers of Competence). The spin-offs are 'incubated' for one to two years in the research institutes from which they emerged, in the ETH area of the TECHNOPARK Zurich, or for a few years in the ETH Innovation and Entrepreneurship Lab (ieLab) before they are able to rent functional and af- fordable laboratory infrastructures in the BIO-TECHNOPARK Schlieren-Zurich, for example. Through close collaboration with real estate partners and government, a nationally and internationally respected science park is emerging in Schlieren in the periphery of Zurich. Through selection, coaching and placement, over 30 successful start-up companies were able to take up residence in Schlieren. Some of them were purchased by large corporations, but are still active in the park. Together with the research groups of the University of Zurich and the University Hospital of Zurich, an innovative mix of totally new and well-established companies, as well as academic research groups, has developed. The life sciences companies alone employ over 500 employees onsite. The BIO-TECHNOPARK helps and supports start-up companies and promotes exchanges between start-ups, established companies and university research groups. Laboratory facilities are rented out by two real estate partners, with the industrial and commercial center Schlieren AG (GHZ) at the forefront, which has made large investments into the development of an exemplary laboratory infrastructure in recent years. The BIO-TECHNOPARK received recognition through successful company sales and collaborations with the pharmaceutical industry. Thus the ETH spin-off Glycart, which specializes in antibody glycosylation, was purchased by Roche in 2005 for CHF 235 million. Eight years later, the new drug discovered by Roche 
Glycart, Gazyva, received the status 'breakthrough therapy' as the first drug ever to receive FDA approval for use in the USA as a treatment for chronic lymphocytic leukemia. After venture capitalists invested more than CHF 80 million, ESBATech, a spin-off of the University of Zurich, was successfully sold to the Novartis company Alcon in 2009. For CHF 600 million, Alcon secured the antibody fragment technology for the development of ophthalmology products. The ESBATech shareholders kept the technologies and products not related to ophthalmology, and they were incorporated into a new company named Delenex Therapeutics. The antibody analogues (DARPins) from the company Molecular Partners are highly sought after. With Janssen Biotech, Allergan and Roche research and development collaborations, the university spin-offs deal value amount to a total of more than CHF 3 billion, not including the possible royalties from sales.

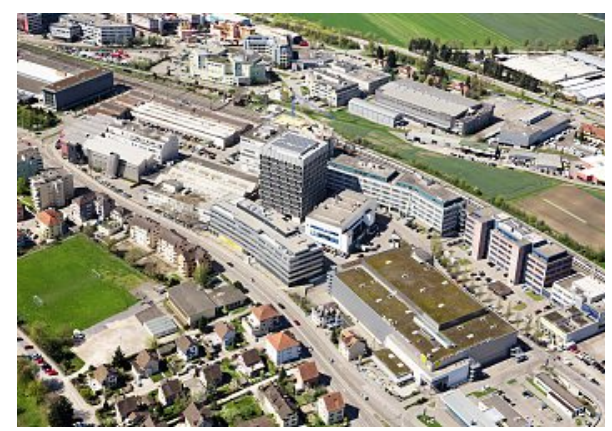

Aerial view of the BIO-TECHNOPARK within the 'Wagi' site with the first of four planned laboratory highrises in the center.

The physical proximity to academic research is important for the spin-offs even after the incubation phase. The University of Zurich's cooperation with the University Hospital of Zurich and the industry in the field of development of new therapies for Alzheimer's disease is a perfect example of efficient, translational medicine. Antibodies discovered by the Division of Psychiatric Research at the university were re-engineered by Neurimmune, a spin-off of the university, and were tested in clinical trials on patients in conjunction with Biogen-Idec, one of the largest biotech companies in the world. The stateof-the-art integrated PET/MR scanner at the University and University Hospital of Zurich play an important role in the evaluation of therapy results. The PET/MR scanner is a combination molecular imaging machine, combining positron emission tomography (PET) and magnetic resonance tomography (MR). The CT/PET/MR center is one of a total of three centers in the world with this new technology, and is located in the BIO-TECHNOPARK, along with the Division of Psychiatric Research and Neurimmune.

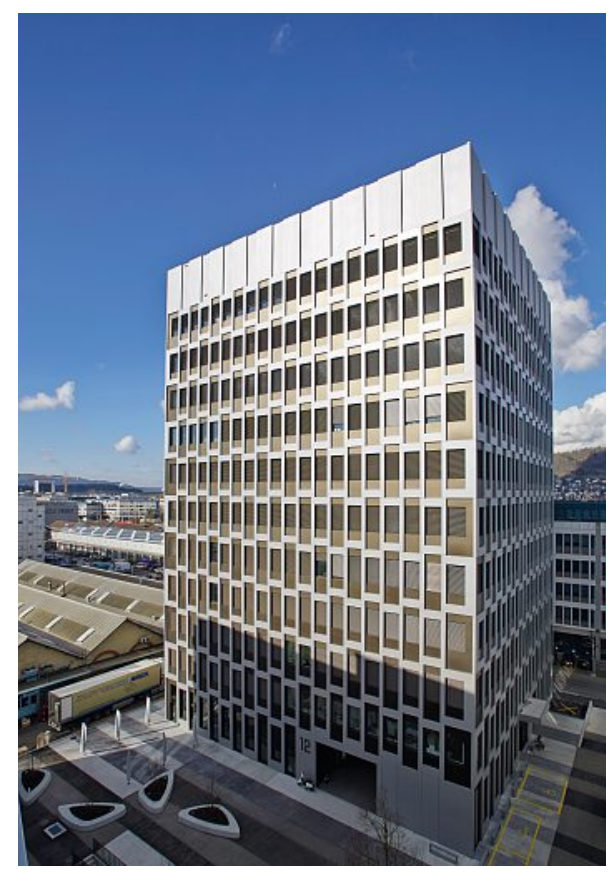

New 11-story laboratory building in the BIOTECHNOPARK.

The start-ups in grow Wädenswil near Zurich also benefit from the close proximity to academia. Grow works closely with the Zurich University of Applied Sciences (ZHAW) which operates the Department of Life Sciences and Facility Management in Wädenswil. ZHAW has excellent infrastructure in these fields and a great deal of highly specialized equipment that is available to the grow tenants by agreement.

The medtech start-ups in the Greater Zurich Area are less concentrated in centers than the biotech start-ups. Concentrations are found in the TECHNOPARKs and, more recently, in Start Smart in Schlieren, a new innovation and business incubator in vicinity to the BIO-TECHNOPARK. Four well-known medtech start-ups have established themselves there: YouRehab, Pearltec, VirtaMed and Aeon Scientific.

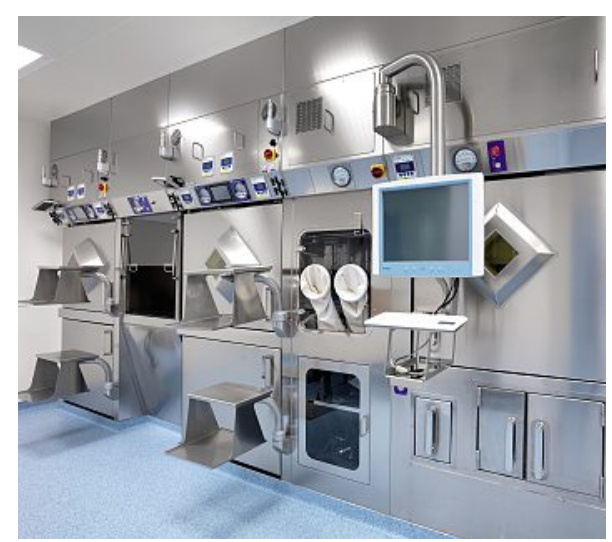

Partial view of a complex manufacturing facility of the University Hospital in the BIOTECHNOPARK with bunkers, clean rooms, sluices and technical areas for the production of radiopharmaceuticals.
Life Science Incubators and

Parks in the Greater Zurich Area

(Agglomeration Zurich and Eastern Switzerland)

- Balgrist Campus

http://www.balgristcampus.ch/

- BIO-TECHNOPARK ${ }^{\circledR}$ SchlierenZurich http://www.bio-technopark.ch/

- ETH Innovation and Entrepreneurship Lab (ieLab) https://www1.ethz.ch/vpf/ielab

- Grow Wädenswil

http://www.grow-waedenswil.ch/

- Start Smart Schlieren

http://www.start-smart-schlieren.ch/

- Swiss Innovation Park Zurich www.innovationspark.zh.ch/

- TECHNOPARK ${ }^{\circledR}$ Zurich http://www.technopark.ch/

- TECHNOPARK ${ }^{\circledR}$ Winterthur http://www.tpw.ch/

Start Smart cannot yet be called an actual medtech cluster because of small size and low synergy potential. Synergies can only be used optimally if common needs can be clearly identified (e.g. joint laboratory infrastructure). In the heterogeneous medtech field this is the case when the stakeholders are active in the same area. For example, YouRehab, along with Dynamic Devices and Hocoma, is integrated into the academic initiative 'Rehabilitation Initiative and Technology Platform Zurich' (RITZ) of the University and ETH Zurich as an 'observer'. Members of this platform are departments, laboratories, hospitals and institutions in Zurich that are active in the field of neurorehabilitation with the goal of using synergies, optimizing knowledge transfers, and stimulating exchanges between neurosciences, engineering and clinical sciences in the fields of research, education and patient care.

Another example of a highly specific cluster is Toolpoint for Lab Science in Hombrechtikon, which combines the knowledge and skills of the laboratory automation industry in Europe. It was founded 11 years ago by companies in the Greater Zurich Area. Toolpoint currently represents 29 members with over 20,000 employees worldwide, reaching sales of nearly USD 5 billion.

In northeast Switzerland, there is a trinational network named BioLAGO. The Life Science Network BioLAGO, funded through public-private partnerships, unites closely cooperating companies and research institutions around Lake Constance in Switzerland, Germany and Austria. The BioLAGO association catalyzes and supports the development of innovative and financially sound industries for the modern life sciences in the international Lake 


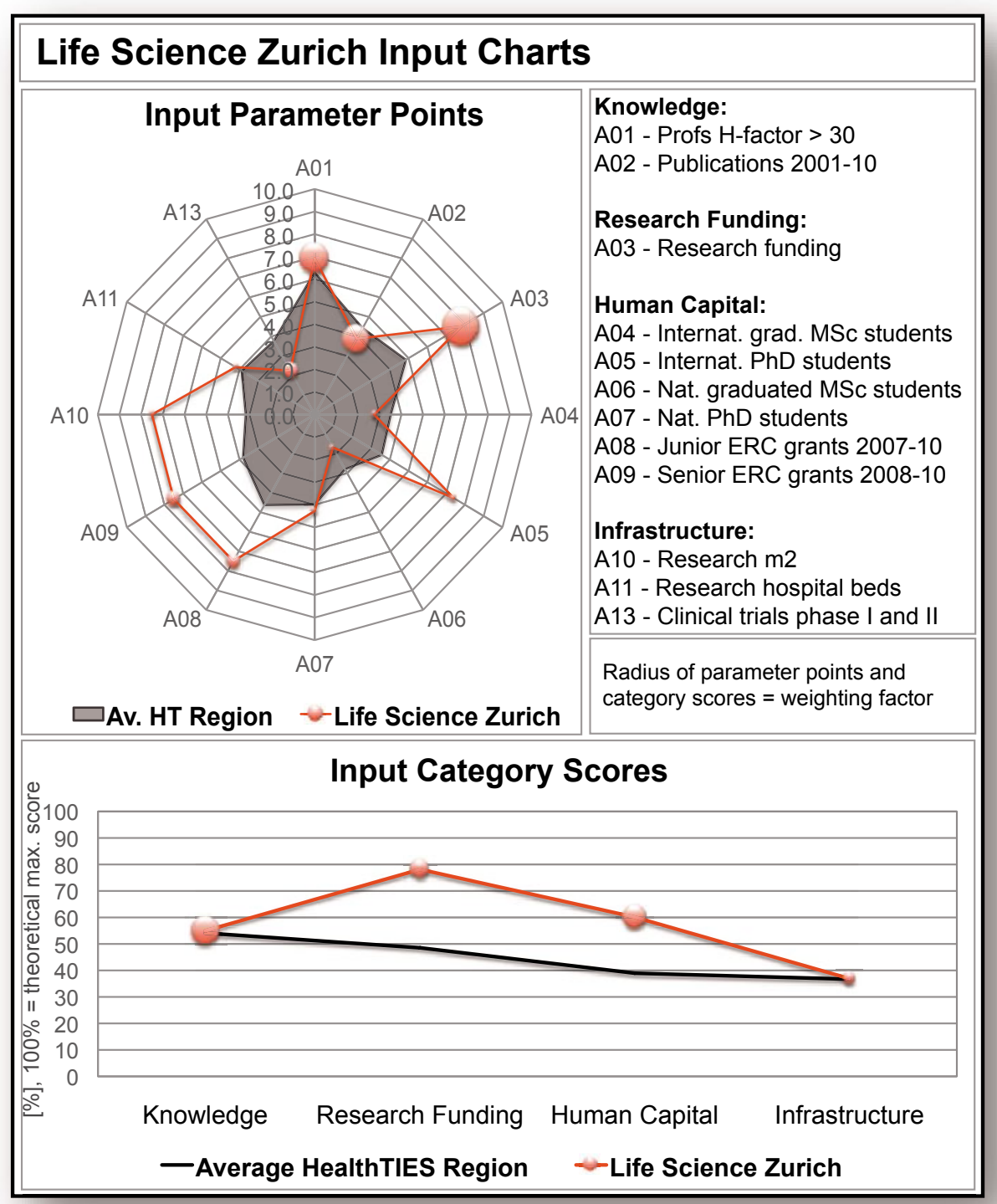

Fig. 1. HealthTIES benchmarks region Zurich, for the field of input. For comparison, the average of the HealthTIES partner regions is given (without the mentoring region Ëszak-Alföld).

Constance area. BioLAGO represents over 5,700 highly skilled jobs. Over 75 member companies finance 5,250 jobs in life sciences in their businesses and represent six research and development-oriented colleges and institutions with approximately 500 scientists. Included among them are the Swiss Federal Laboratories for Materials Science and Technology (Empa St. Gallen) and the University for Applied Sciences (FHS St. Gallen).

The Greater Zurich Area also has a life sciences network which combines academia, industry and government authorities (Triple Helix). In the fall of 2001, the University of Zurich and the ETH Zurich started a joint initiative with the project Life Science Zurich to link the research in this field together more strongly. Other successful areas of activity have been added to the original task of networking and strengthening communi-

\section{Comparison of the Zurich Cluster and other European Technology Parks[4]}

The institutions of the Life Science Zurich Business Network, along with the ETH and the University of Zurich, have combined into a consortium with the regions Delft/Leiden (Holland), Oxford/ Thames Valley (England), Catalonia (Spain) and Ëszak-Alföld (Hungary) as part of the EU project HealthTIES (Health Technology Innovation and Economic Success) which was supported by the EU Commission from 2010 to 2013 with about EUR 2.1 million. ${ }^{[5]}$ The goal was to develop a foundation for new strategies and tools to promote innovation, accelerate innovation cycles and shorten the implementation time for marketable products and services in the health industry. Innovations in the field of life sciences often take place in regional and technology-driven networks in a complex interplay of various stakeholders from academic and clinical research, biotech, medtech and the pharmaceuticals industry. Government agents have an important influence on the structure and surrounding conditions of such clusters. But what are the success factors of competitive clusters? How are the innovation ecosystems structured? What are the differences between different regions? The core of the work of the HealthTIES consortium was the development and implementation of an in-depth comparative analysis of the participating regions in terms of a best practices comparison and benchmarking to answer the above questions. A set of indicators was developed for the multidimensional comparison that includes the entire process of research and development and allows a useful characterization of the innovation architecture. The data compiled in all regions allows deep insight into the morphology of the partner regions and shows strengths and weaknesses. Beyond the project results, an important use of HealthTIES was in allowing tighter networking between the partner regions, but also between stakeholders within the individual regions. In this international comparison, the region of Zurich performs well: It has an extremely well-arranged academic system. In particular, this refers to high-quality academic research, the comparatively high investment in research and the Zurich universities' ability to attract excellent researchers. The only indicator that was greatly below average was the number of clinical trials in phases I and II. But this result is not limited only to the Greater Zurich Area. According to Interpharma, the number of clinical trials (phases I to IV) in Switzerland almost halved to a total of 205 in $2013,{ }^{[6]}$ a result that is a cause for concern, given the importance of the 


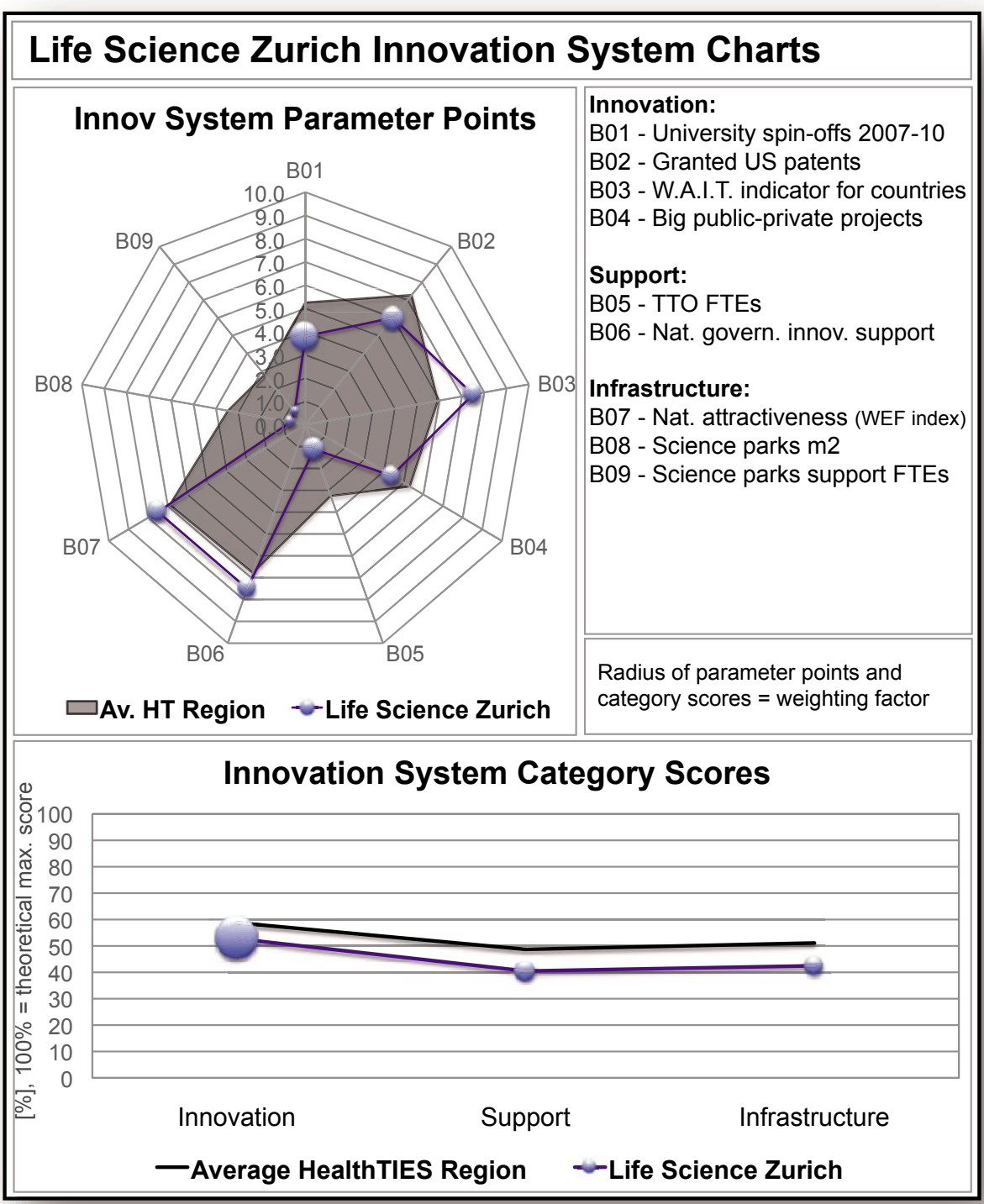

Fig. 2. HealthTIES benchmarks region Zurich, for the field of innovation systems. For comparison, the average of the HealthTIES partner regions is given (without the mentoring region Ëszak-Alföld).

early research phases for future products and the attractiveness of the research location. Currently, the indicators show that the region of Zurich is well-positioned in the output and products area, with an aboveaverage number of biotech companies and a large number of products on the market. In addition to the weaknesses in the field of clinical research, there is also the potential for improvement at the juncture between academia and industry, where, in Zurich, for example, the technology transfer sites and the staff of the science parks are seriously underfunded by comparison. A focus on the efficient translation of academic performance into products is worthwhile to enable a continued strong position in the region. With the methodology developed in HealthTIES (Figs 1-3), an applicable tool beyond the scope of the project was created to analyze the innovation architecture of the regions.

\section{Future Development}

The offerings for life sciences in the Greater Zurich Area will be expanded in the coming years at the infrastructure level. In addition to the 11-story laboratory highrise opened in 2014 in the BIOTECHNOPARK, three more are planned to create space for new and expanding life sciences companies and research organizations. A campus-like atmosphere will be created by decompressing and opening up the area to the surrounding neighborhood.

Balgrist Campus, a research and development building for musculoskeletal medicine, is under construction in the southeast side of Zurich in the lakeside area of University Clinic Balgrist. The building should be ready in the fall of 2015 . It will benefit from the close proximity to patients, clinical doctors, researchers and engineers, as well as direct relationships with the University and ETH Zurich. The medtech industry and start-ups will also be present in the research and development building. The Balgrist Campus is privately funded, like the buildings in the BIO-TECHNOPARK.

As one of two national hubs, about 70 square hectares of land at the top of Dübendorf airfield near Zurich is designated for the planned Swiss Innovation Park. The field will be developed in stages according to demand, where an area of 10-25 hectares is targeted in the first stage of expansion. That corresponds to an area of about 35 soccer fields. The park will be merged with Dübendorf, will have urban structures for living, working and residences, and will be naturally embedded into the existing infrastructures. It is not yet certain to what extent the innovation park will be thematically focused. Life Sciences $\&$ Quality of Life was determined as one of the possible theme directions for the innovation park, and is defined as follows: technological expertise that contributes to the recovery, maintenance and increased comfort of life.

How strong the life sciences industry in the Greater Zurich Area will become in the future is strongly dependent on the worldwide economic situation and the political environment. Problem areas such as financing gaps for start-ups in the early phases, the decline in the number of clinical trials, difficult access to European research projects, or the recruitment limitation for the European workforce, just to name a few, must be approached jointly and throughout Switzerland in terms of 'One Nation, One Life Science Cluster'. 


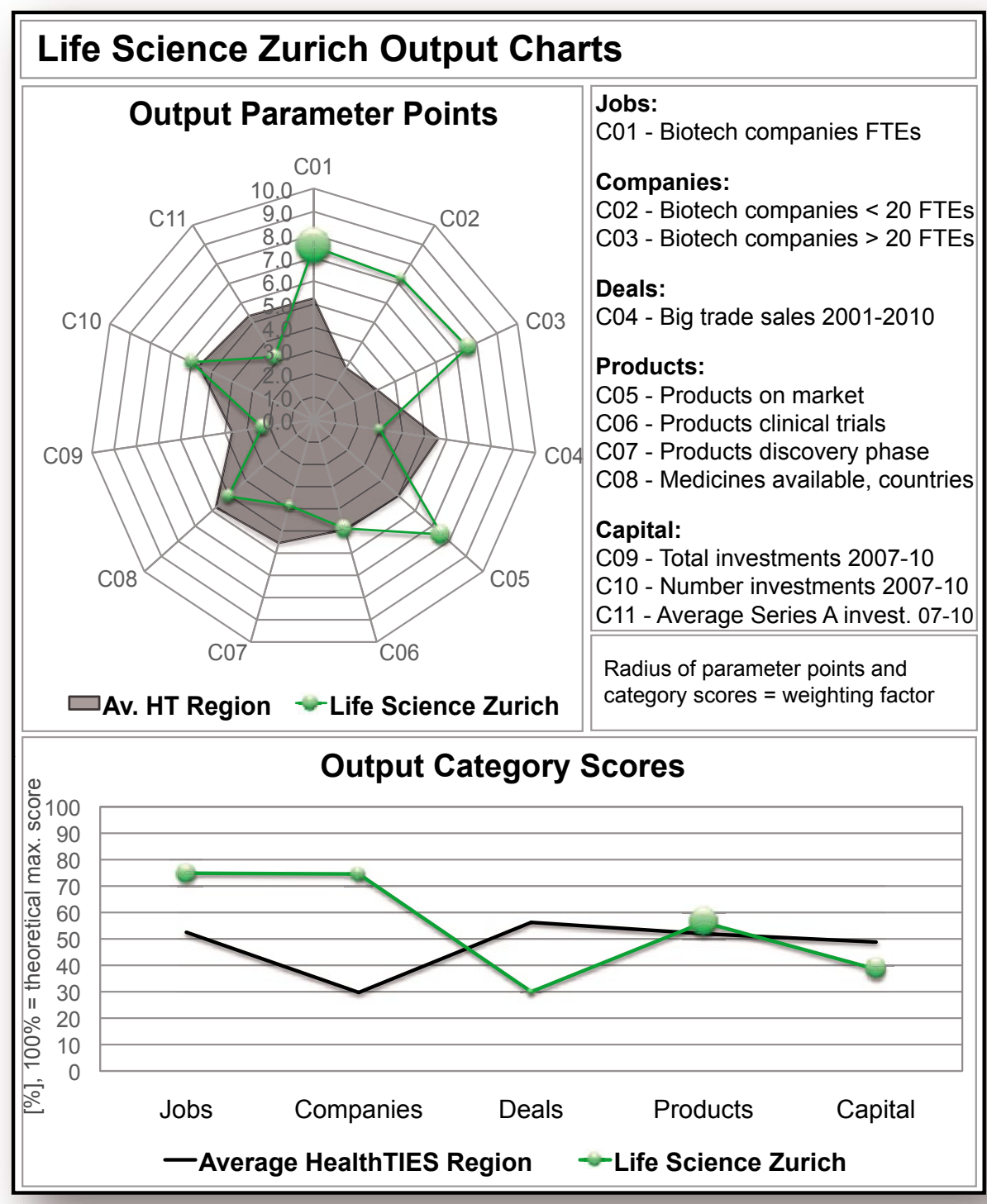

Fig. 3. HealthTIES benchmarks region Zurich, for the fields of output. For comparison, the average of the HealthTIES partner regions is given (without the mentoring region Ëszak-Alföld).
Received: September 18, 2014

[1] Canton of Zurich, Department for Economic Affairs, Office for Economy and Labour Cluster-Report 2011-2013, 34.

[2] Swiss Life Sciences Database, http://www. swisslifesciences.ch/

[3] Academic Ranking of World Universities 2014, http://www.shanghairanking.com/

[4] D. Steiger, M. Jenni, Schweizer Krebsbulletin 2014, $1,26$.

[5] The research leading to these results has received funding from the European Union's Seventh Framework Programme (FP7/20072013) under grant agreement no. 265550.

[6] Interpharma, Pharmaceuticals Market Switzerland 2014. 